\title{
Confocal laser endomicroscopy for diagnosis of Barrett's esophagus
}

\author{
Helmut Neumann ${ }^{1}$, Cord Langner ${ }^{2}$, Markus F. Neurath ${ }^{1}$ and Michael Vieth ${ }^{3}$ * \\ 1 Department of Medicine I, University of Erlangen-Nuremberg, Erlangen, Germany \\ 2 Institute of Pathology, Medical University Graz, Graz, Austria \\ ${ }^{3}$ Institute of Pathology, Klinikum Bayreuth, Bayreuth, Germany
}

Edited by:

Alessandro Repici, Istituto Clinico

Humanitas, Italy

Reviewed by:

Mirko Omejc, University Medical

Centre Ljubljana, Slovenia

Parham Minoo, University of

California San Diego, USA

*Correspondence:

Michael Vieth, Institut für Pathologie,

Klinikum Bayreuth $\mathrm{GmbH}$,

Preuschwitzer Str. 101, 95445

Bayreuth, Germany.

e-mail: pathologie@klinikum

bayreuth.de
Barrett's esophagus (BE) is established as a premalignant condition in the distal esophagus. Current surveillance guidelines recommend random biopsies every $1-2 \mathrm{~cm}$ at intervals of 3-5years. Advanced endoscopic imaging of BE underwent several technical revolutions within the last decade including broad-field (red-flag) techniques (e.g., chromoendoscopy) and small-field techniques with confocal laser endomicroscopy (CLE) at the forefront. In this review we will focus on advanced endoscopic imaging using CLE for the diagnosis and characterization of BE and associated neoplasia. In addition, we will critically discuss the technique of CLE and provide some tricks and hints for the daily routine practice of CLE for diagnosis of $\mathrm{BE}$.

Keywords: endomicroscopy, Barrett, cancer, CLE, advanced imgaging, fluorescein, dysplasia

\section{INTRODUCTION}

Barrett's esophagus (BE) is a metaplastic condition which is characterized by the replacement of normal squamous epithelium into columnar (intestinal) epithelium within the distal esophagus. There is an ongoing discussion whether the diagnosis of $\mathrm{BE}$ needs histologically proven goblet cells as a prerequisite. At this time it is also unclear how to proceed clinically with cases without such goblet cells. The prevalence of BE still remains unknown. It is estimated that $\mathrm{BE}$ affects about $1-3 \%$ of the general population, although in autopsy studies the prevalence was reported to be only $0.4-0.9 \%$ (Winters et al., 1987; Cameron et al., 1990; Cameron, 1993; Ormsby et al., 2000; Vieth and Stolte, 2002). Probably these numbers are even lower according to the above mentioned discussion.

Gastroesophageal reflux disease (GERD) has been identified as the major risk factor for the development of $\mathrm{BE}$, while advanced age ( $>60$ years), obesity, male sex, and white race were additionally considered to be risk factors of BE (Gaddam and Sharma, 2010).

By itself, $\mathrm{BE}$ is considered to be the key risk factor for the development of esophageal adenocarcinoma (EAC). Compared to the general population, patients with $\mathrm{BE}$ are at a 30 - to 50 -fold increased risk for the development of EAC (Tytgat, 1995; Vieth et al., 2004). In this context it has been suggested that specialized intestinal metaplasia with goblet cells is the predominant preneoplastic condition which progressively develops to low-grade intraepithelial neoplasia (LGIEN), to high-grade intraepithelial

\footnotetext{
Abbreviations: EAC, esophageal adenocarcinoma; FICE, Fujinon intelligent color enhancement; HD-WLE, high-definition white light endoscopy; HGIEN, highgrade intraepithelial neoplasia; iCLE, integrated confocal laser endomicroscopy; LGIEN, low-grade intraepithelial neoplasia; NBI, narrow band imaging; pCLE, probe-based confocal laser endomicroscopy.
}

neoplasia (HGIEN), and than to EAC (Reid et al., 1993; Vieth, 2007). Nevertheless, recent data indicate that even patients without goblet cells in the columnar lining at the distal esophagus show the same chromosomal and DNA content abnormalities and genetic instabilities compared to goblet cell containing esophageal metaplasia (Liu et al., 2009; Vieth and Barr, 2009). Moreover, one recent multicenter study including 210 patients with BE and LGIEN with a mean follow-up of 6.2 years demonstrated that patients with $\mathrm{BE}$ and LGIEN have a low annual incidence of about $0.44 \%$ /year of EAC which is similar to patients with non-dysplastic BE. In addition, no "convincing" risk factors for the progression of LGIEN to EAC could be found other than LGIEN itself preferably confirmed by a second opinion (Wani et al., 2011).

Therefore, all metaplasia in the columnar lined esophagus may already be on the path to cancer and need distinct examination by the exploiting team of an endoscopist and histopathologist.

Hence, current guidelines from most gastroenterological societies worldwide recommend endoscopic screening for the detection of BE for patients with chronic GERD. If a BE-segment is identified four-quadrant random biopsies at $2-\mathrm{cm}$ intervals are recommended in patients without suspicion of HGIEN or at $1-\mathrm{cm}$ intervals in patients with suspected HGIEN of metaplastic tissue supplemented with targeted biopsies of grossly suspicious areas according to the Seattle protocol (Peters et al., 2008).

While the presence of intraepithelial neoplasia or early adenocarcinoma within a BE-segment may occur patchy, standard white light endoscopy with random biopsy may miss a certain quantum of lesions. Moreover, the random biopsy protocol has several limitations, as it is time-consuming, expensive, and prone to sampling error because less than $10 \%$ of the surface area is sampled (Hwang, 2009). 
Taken into account the tremendous increasing incidence of BE (about $350 \%$ over the past 20 years), the need for improved imaging techniques for diagnosis and characterization of $\mathrm{BE}$ becomes obvious.

Indeed, endoscopic imaging of BE has seen several technical revolutions within the last two decades. These new imaging techniques can be subdivided into broad-field techniques ("redflag") which allow the examination of the whole luminal surface area and small-field imaging techniques which provide an optical biopsy of the tissue. Red-flag techniques include both, dye-based (e.g., methylene blue) and dye-less chromoendoscopy (narrow band imaging, NBI; FICE; i-scan) (Figure 1) and magnification endoscopy while small-field imaging techniques include endocytoscopy and confocal laser endomicroscopy (CLE; Neumann et al., 2012).

Here, we will focus on advanced endoscopic imaging using CLE for the diagnosis and characterization of $\mathrm{BE}$ and associated neoplasia. In addition, we will critically discuss the technique of CLE and provide some tricks and hints for the daily routine practice of CLE for diagnosis of BE.

\section{TECHNICAL BACKGROUND}

Confocal laser endomicroscopy is based on the principle of tissue illumination with a blue laser light (488 nm wave length) after application of fluorescence agents which can either be applied topically (e.g., acriflavine hydrochloride, cresyl violet) or systemically (fluorescein sodium; Neumann et al., 2010).

Up to now, only the topical applied fluorescence agents enabled imaging of cell nuclei which is essential to differentiate between LGIEN and HGIEN according to the Vienna classification of gastrointestinal neoplasia (Schlemper et al., 2000). While acriflavine accumulates in nuclei a potential mutagenic risk of this drug is discussed. Cresyl violet was recently introduced as an alternative fluorescence agent for confocal imaging which provides cytoplasmic enrichment and thereby

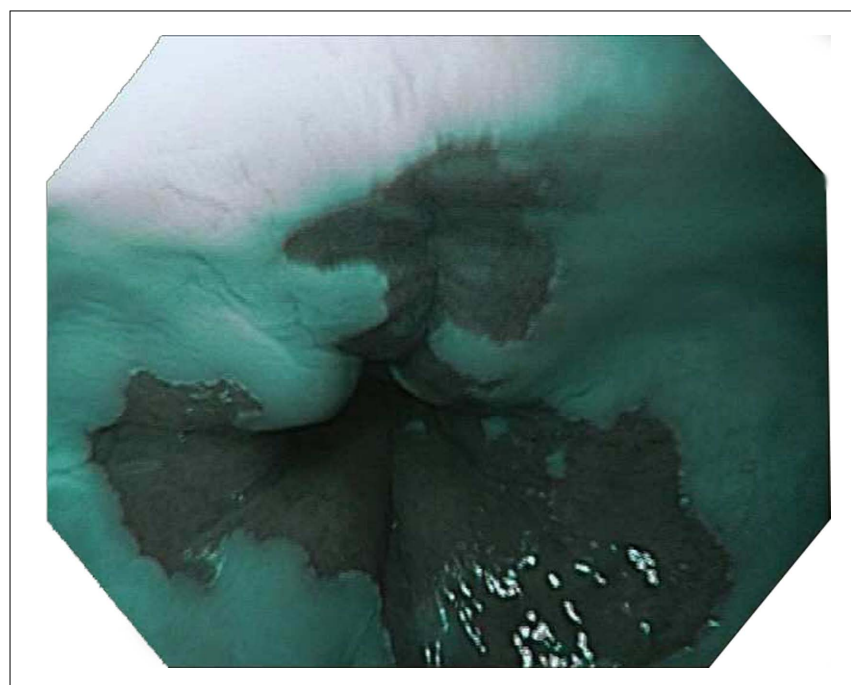

FIGURE 1 |Typical endoscopic appearance of Barrett's esophagus imaged using narrow band imaging (NBI) with single mucosal tears proximal to the esophagogastric junction. negative visualization of nuclear morphology (Goetz et al., 2009).

Nevertheless, the most widely used fluorescent agent to date is intravenous fluorescein (mostly $3-5 \mathrm{ml}$ of a $10 \%$ solution). Fluorescein is a non-toxic agent and FDA-cleared for diagnostic angiography of the retina for years. Importantly, as fluorescein is a non-selective vascular contrast agent, only architectural details could be imaged. The safety of intravenous fluorescein for confocal imaging was recently demonstrated in a large multicenter study, including 16 centers and nearly 2300 gastrointestinal endomicroscopy procedures (Wallace et al., 2010a). In this study no serious adverse events were reported. Mild adverse events occurred in $1.4 \%$ of individuals, including transient hypotension without shock $(0.5 \%)$, nausea $(0.39 \%)$, injection site erythema $(0.35 \%)$, self-limited diffuse rash $(0.04 \%)$, and mild epigastric pain $(0.09 \%)$.

Currently, two different endomicroscopy systems are commercially available (Neumann et al., 2010). One is integrated into the distal tip of a standard, high-resolution endoscope (iCLE; Pentax, Tokyo, Japan) (Figure 2) and one is probe-based (pCLE; Cellvizio, Mauna Kea Technologies, Paris, France) (Figure 3) capable of passage through the accessory channel of nearly any endoscope. Both systems use an incident $488 \mathrm{~nm}$ wavelength laser and allow both, real-time observation and post hoc analysis of the tissue at about 1000-fold magnification.

The iCLE-system has an imaging plane depth ranging from 0 to $250 \mu \mathrm{m}$. The optical slice thickness is $7 \mu \mathrm{m}$, with lateral and axial resolutions of $0.7 \mu \mathrm{m}$. The observer can manually adjust the laser power output at the tissue surface ranging from 0 to $1000 \mu \mathrm{W}$. Confocal images are captured at a manually adjustable frame rate of $1.6 \mathrm{images} / \mathrm{s}$ at a resolution of $1024 \times 512$ pixels, or at $0.8 \mathrm{images} / \mathrm{s}$ at a resolution of $1024 \times 1024$ pixels with a confocal image field of view of $475 \times 475 \mu \mathrm{m}$.

The pCLE-system offers different endomicroscopy-probes for luminal and non-luminal confocal exploration. Probes for endomicroscopy during esophagogastroduodenoscopy require accessory channels of $2.8 \mathrm{~mm}$ and have a working length of $3 \mathrm{~m}$. Lateral resolution is $1 \mu \mathrm{m}$ which constitutes to a $43 \%$ decrease in resolution compared to the iCLE-system (Bisschops and Bergman, 2010). Confocal images are streamed at a video frame rate of 12 frames/s. The system has a fixed laser power and a fixed image plane depth of 55-65 $\mu \mathrm{m}$.

In order to achieve complete agreement between confocal diagnosis and biopsy acquisition for subsequent histopathological diagnosis one has to keep in mind that the working channel of the iCLE-system is located $5 \mathrm{~mm}$ to the right of the confocal lens. Therefore, when suction is applied to the mucosa, the resulting intramucosal hemorrhage is located $5 \mathrm{~mm}$ to the right from the area which has been evaluated using iCLE. For pCLE mild pressure is performed with the confocal probe to the tissue and the resulting reddish mucosa can guide subsequent biopsy acquisition. Moreover, we recommend attaching a clear cap at the distal tip of the endoscope for pCLE-imaging as motion artifacts substantially impede confocal imaging at the squamocolumnar junction. Even administration of antispasmodic agents (e.g., Buscopan) may help to stabilize the confocal probe for subsequent high-magnification imaging of the mucosa. 


\section{ENDOMICROSCOPY OF BARRETT'S ESOPHAGUS}

The first study of confocal imaging for diagnosis of BE was introduced in 2006 and included 63 patients (Kiesslich et al., 2006). In this pioneer study by Kiesslich and coworkers iCLE was proven to reliable identify different epithelial cell types at the squamocolumnar junction, including squamous epithelium, gastric epithelium, and specialized intestinal epithelium containing goblet cells. Goblet cells could easily be identified as CLE displayed the mucin of goblet cells as very dark oval to round appearing structures within the columnar lined epithelium. In patients with BE-associated neoplasia CLE imaged large, irregular sheets of black cells which showed a marked dark abrupt contrast to the surrounding tissue and loss of regular architecture with irregular lumina of different diameter. The dark contrast is probably due to the lower $\mathrm{pH}$ within neoplastic cells. Additionally, the brightness of the

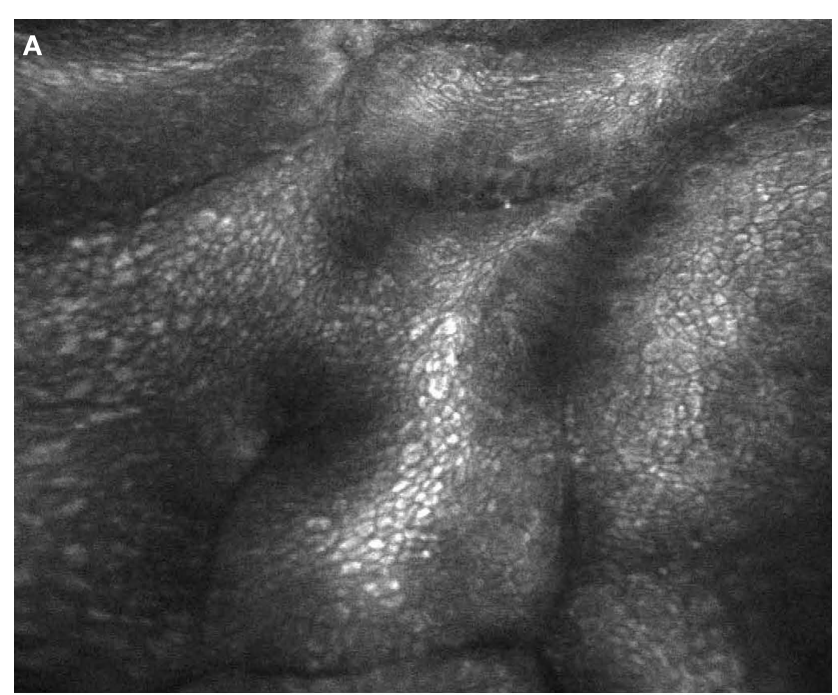

B

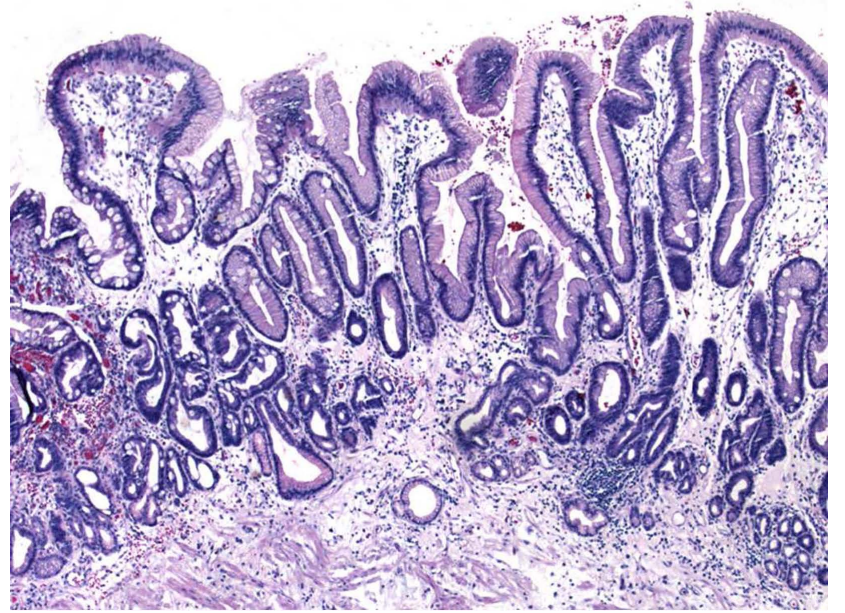

FIGURE 2 | (A) Confocal laser endomicroscopy using the integrated device (iCLE). Regular shaped columnar lined epithelium with goblet cells (arrows) is clearly visible. (B) Histological image of Barrett's epithelium with numerous typical goblet cells that replaced the regular squamous epithelium in the distal esophagus (H\&E 200×). lamina propria became heterogeneous according to an increased and irregular vasculature with fluorescein leakage. Based on these parameters the authors proposed a Confocal BE-classification to predict histopathology in the distal esophagus. In vivo imaging could predict BE and associated neoplasia with a sensitivity of 98 and $93 \%$, specificity of 94 and $98 \%$, and accuracy of $97 \%$, respectively. Of note, most of the patients with neoplasia $(80 \%)$ had mucosal irregularities or focal lesions that could be detected by high-resolution white light endoscopy.

This is of particular importance when taken into account the data of Bajbouj et al. (2010) where pCLE was compared with a standard four-quadrant biopsy protocol for the evaluation of neoplasia in BE. In this multicenter study a total of 670 pCLEmatched biopsies were obtained from 68 patients. The authors studied the diagnostic accuracy of pCLE for real-time diagnosis during ongoing endoscopy and in a blinded fashion by scoring the confocal images without having the endoscopic image available.
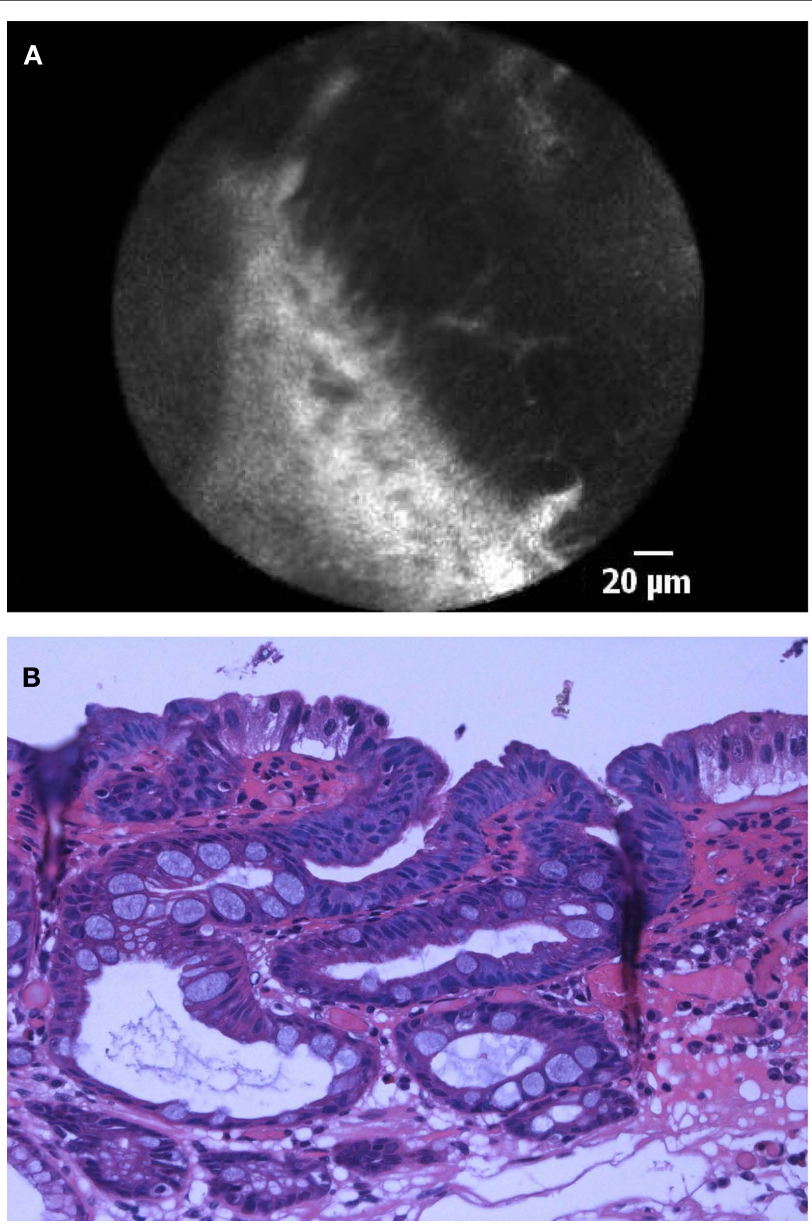

FIGURE 3 | (A) pCLE image of high-grade intraepithelial neoplasia in patient with BE. Confocal imaging demonstrated dark, irregularly thickened epithelial cells, and dilated irregular vessels. (B) Histological image of Barrett's esophagus with high-grade intraepithelial neoplasia. Cytological and architectural atypia is visible. The nuclei are larger in size with increased chromaticity, and loss of cell polarity. Architectural distortion is characterized by a back-to-back crypt pattern and focal cribriform areas. 
The study demonstrates that correct image interpretation depends on simultaneous elucidation of endoscopic and confocal images, as on a per-patient basis, a significant decrease in specificity from 95 to $59 \%$ was observed when investigators were blinded to the endoscopic findings.

Therefore, simultaneous interpretation of macroscopic and microscopic images is essential as "The eye sees only what the mind is prepared to comprehend" (Henri Bergson 1859-1941, French philosopher).

In another prospective two-center trial 38 consecutive patients with $\mathrm{BE}$ were examined with standard high-resolution white light endoscopy and pCLE (Pohl et al., 2008). In a per-biopsy analysis, sensitivity and specificity for two independent investigators were 75 and $89 \%$, and 75 and $91 \%$, respectively, translating at best into a positive predictive value of $44 \%$ and a negative predictive value of 99\% (Pohl et al., 2008).

One prospective, randomized, double-blind, controlled, crossover trial at a tertiary referral center evaluated whether iCLE with targeted mucosal biopsy improves the diagnostic yield of endoscopically inapparent, BE-associated neoplasia compared to standard endoscopy with a four-quadrant, random biopsy protocol (Dunbar et al., 2009). Overall, 39 patients completed the study protocol. It was found that iCLE with targeted biopsy almost doubled the diagnostic yield for neoplasia (34 vs. 17\%) and was equivalent to the standard protocol for the final diagnosis of neoplasia. Moreover, two thirds of patients in the surveillance group did not need any mucosal biopsies at all. Another important lesson from the study is that there were two patients with HGIEN only identified using iCLE, whereas another two patients with HGIEN were only identified by standard white light endoscopy with random biopsies. This illustrates the limitation of CLE, which is a small-field imaging technique, therefore prone to sampling error.

Wallace et al. (2010b) determined the preliminary accuracy and interobserver agreement of pCLE in BE using a prospective, double-blind review of confocal images of BE after a training set of 20 images with known histology was reviewed to standardize image interpretation. The sensitivity for the diagnosis of neoplasia for the 11 attending endoscopists from different international centers was $88 \%$, and the specificity was $96 \%$ with a substantial agreement on the pCLE diagnosis $(86 \%, \kappa 0.72 ; 95 \%$ confidence interval, 0.58-0.86). These results suggest that pCLE for the diagnosis of neoplasia in BE has a very high accuracy and reliability and can relatively easy be learned and used (Figure 4).

Very recently the results of a large prospective international multicenter study on BE including five tertiary referral centers were presented (Sharma et al., 2011). In this study, 101 consecutive patients with $\mathrm{BE}$ were examined by high-definition white light endoscopy (HD-WLE), NBI, and pCLE, and the findings were recorded before biopsy samples were obtained. The sensitivity and specificity for HD-WLE were 34 and 93\%, respectively, compared with 68 and $88 \%$, respectively, for HD-WLE or pCLE which was statistically significant different. The sensitivity and specificity for HD-WLE or NBI were 45 and $88 \%$, respectively, compared with 76 and $84 \%$, respectively, for HD-WLE, NBI, or pCLE which again was statistically significant. Therefore, pCLE combined with HDWLE significantly improved the ability to detect neoplasia in $\mathrm{BE}$ patients compared to HD-WLE alone.
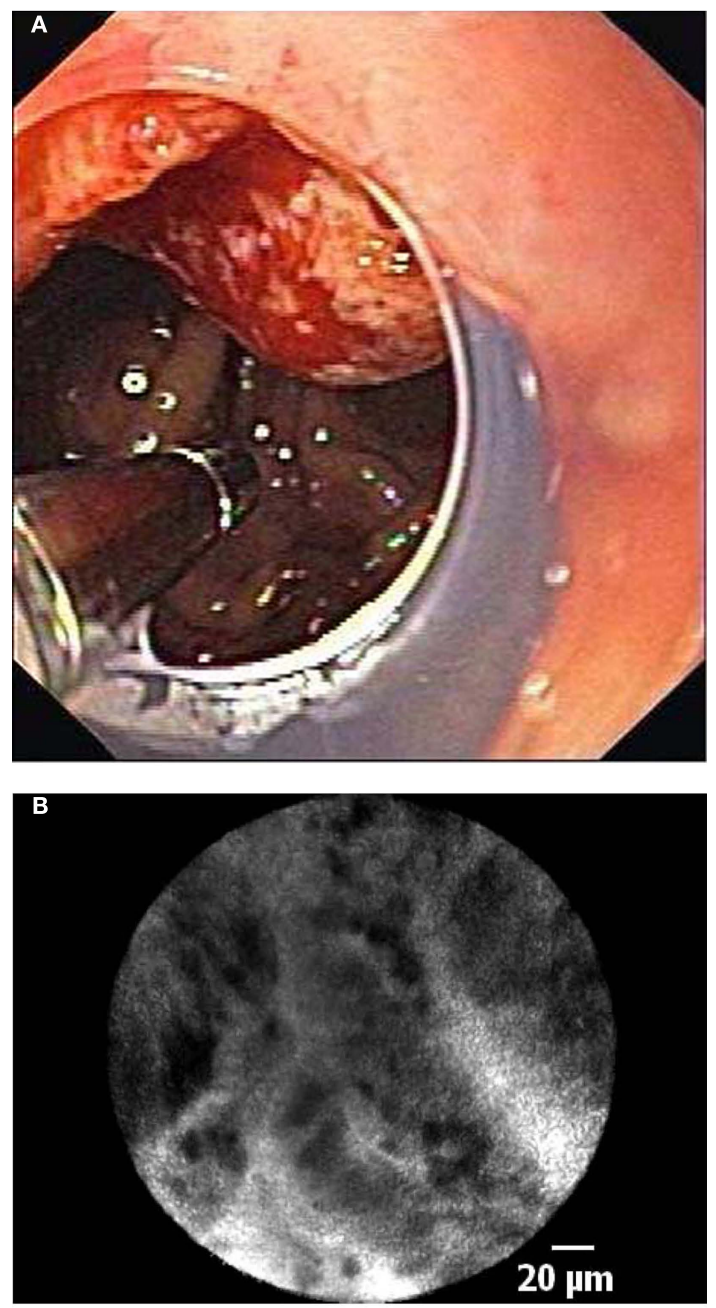

C

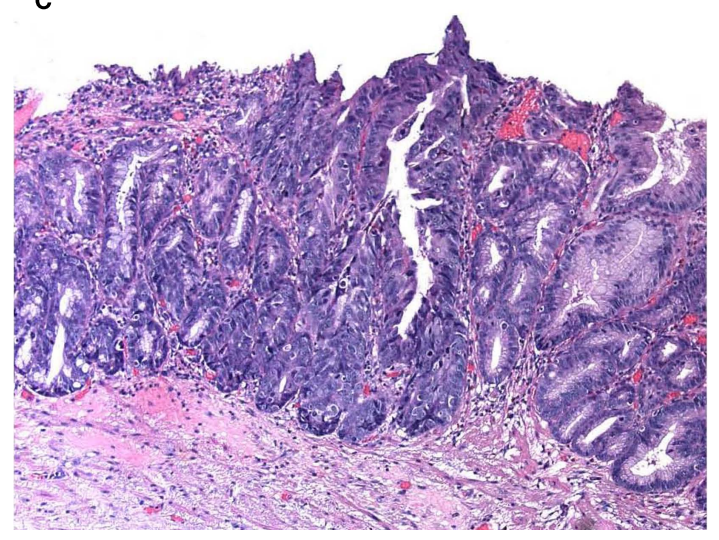

FIGURE 4 | (A) In vivo endomicroscopy of advanced esophageal adenocarcinoma in patient with long-segment Barrett's esophagus using the probe-based device (pCLE). Note the clear cap at the distal tip of the endoscope to avoid motion artifacts. (B) Corresponding endomicroscopic appearance of adenocarcinoma in a patient with Barrett's esophagus. Irregular, dark cells with high contrast to the surrounding tissue indicate advanced neoplasia. (C) Histological image of moderately differentiated adenocarcinoma in a patient with Barrett's esophagus with loss of regular architecture and superficial erosion. 
In order to discriminate new diagnostic criteria for dysplastic BE-tissue again the group from Kansas City evaluated multiple pCLE criteria including the epithelial surface, epithelial cell, and gland morphology (Gaddam et al., 2011). By using these criteria the overall accuracy in diagnosing dysplasia was $82 \%$. Interestingly, no differences in image interpretation were observed between experts and non-experts in confocal imaging. More importantly, after a structured teaching session, accuracy, and agreement between experienced and non-experienced observers was not different, suggesting a short learning curve of image interpretation.

Recently, a consensus of pCLE users for standardization of image criteria was introduced. The so called "Miami classification" described substantial parameters to differentiate between normal squamous epithelium, non-dysplastic Barrett's epithelium, HGIEN, and adenocarcinoma in BE (Wallace et al., 2011). Following this classification, normal squamous epithelium is characterized by flat cells without crypts or villi and bright vessels within papillae, representing the intra papillary capillary loops. Non-dysplastic BE showed uniform villiform architecture with columnar cells and dark appearing goblet cells. In contrast, pCLE images demonstrating villiform structures and dark, irregularly thickened epithelial borders and dilated irregular vessels were specific for BE with HGIEN. Adenocarcinoma in BE was characterized by disorganized or a completely loss of villiform structures and crypts, dark columnar cells, and dilated irregular vessels.

\section{SUMMARY}

Barrett's esophagus is a premalignant condition as a sequel of long standing GERD. Therefore, surveillance is of paramount importance. Nevertheless, the patchy and inhomogeneous distribution of intraepithelial neoplasia within a Barrett's segment makes a correct diagnosis often difficult. Columnar lined epithelium is often a mosaic of different cell types consisting of junctional and cardiac epithelium, specialized intestinal metaplasia with or without

\section{REFERENCES}

Bajbouj, M., Vieth, M., Rösch, T., Miehlke, S., Becker, V., Anders, M., Pohl, H., Madisch, A., Schuster, T., Schmid, R. M., and Meining, A. (2010). Probe-based confocal laser endomicroscopy compared with standard four-quadrant biopsy for evaluation of neoplasia in Barrett's esophagus. Endoscopy 42, 435-440.

Bisschops, R., and Bergman, J. (2010). Probe-based confocal laser endomicroscopy: scientific toy or clinical tool? Endoscopy 42, 487-489.

Cameron, A. J. (1993). Epidemiologic studies and the development of Barrett's esophagus. Endoscopy 25, 635-636.

Cameron, A. J., Zinsmeister, A. R., Ballard, D. J., and Carney, J. A. (1990). Prevalence of columnarlined (Barrett's) esophagus. Comparison of population-based clinical and autopsy findings. Gastroenterology 99, 918-922.
Dunbar, K. B., Okolo, P. III, Montgomery, E., and Canto, M. I. (2009). Confocal laser endomicroscopy in Barrett's esophagus and endoscopically inapparent Barrett's neoplasia: a prospective, randomized, doubleblind, controlled, crossover trial. Gastrointest. Endosc. 70, 645-654.

Gaddam, S., Mathur, S. C., Singh, M., Arora, J., Wani, S. B., Gupta, N., Overhiser, A., Rastogi, A., Singh, V., Desai, N., Hall, S. B., Bansal, A., and Sharma, P. (2011). Novel probe-based confocal laser endomicroscopy criteria and interobserver agreement for the detection of dysplasia in Barrett's esophagus. Am. J. Gastroenterol. 106, 1961-1969.

Gaddam, S., and Sharma, P. (2010). Advances in endoscopic diagnosis and treatment of Barrett's esophagus. J. Dig. Dis. 11, 323-333.

Goetz, M., Toermer, T., Vieth, M., Dunbar, K., Hoffman, A., Galle, P. R., Neurath, M. F., Delaney, P., and Kiesslich, R. (2009). Simultaneous

intraepithelial neoplasia. Random biopsies are prone to sampling error and may miss a quantum of lesions. Besides, they are cost expensive and may even result in severe complications (Vieth and Stolte, 2000; Mannath et al., 2010). Enhanced endoscopic imaging techniques seem to have overcome these limitations and are therefore highly recommended to improve diagnostic outcome of BE patients. CLE allows real-time in vivo assessment of architectural details at a comparable quality to standard histopathology. This is the base for saving time and thus money due to possible immediate further interventional steps such as local endoscopic resection in case of any suspicious lesions. Various large and multicenter studies have shown a substantial accuracy of CLE for diagnosis of $\mathrm{BE}$ and associated neoplasia. Therefore, this new imaging technique offers the potential of enhanced endoscopic imaging of $\mathrm{BE}$ for better surveillance and even to guide further endoscopic management (Leung et al., 2009). Moreover, CLE has great future potential in the assessment of BE as far as further improvement of the technique, which allows visualization of submucosal structures and new dye agents which allow specific visualization of cellular features, become available. This will allow a more precise risk stratification and triage of the patients: surgery vs. local endoscopic resection. Even more, molecular imaging with specific fluorescence-labeled probes against Barrett's epithelium may enhance current concepts of Barrett's management. Nevertheless, despite these exiting ongoing developments and even from a medical-legal point of view more data are needed to justify this approach in the clinical setting. In many countries a diagnosis made on base of tissue (virtual or histologically) is limited to board-certified pathologists also for legal reasons. Regulations may need revision according to these newly available techniques. Up to now, four-quadrant random biopsies according to the Seattle protocol still remained the gold standard for diagnosis and characterization of BE. More relevant and thus localized so called smarter biopsies due to the new imaging techniques may simplify and improve surveillance and planning of interventions.

confocal laser endomicroscopy and chromoendoscopy with topical cresyl violet. Gastrointest. Endosc. 70, 959-968.

Hwang, J. H. (2009). To perform a biopsy or not to perform a biopsy? Does confocal endomicroscopy provide the answer for surveillance in Barrett's esophagus? Gastrointest. Endosc. 70, 655-657.

Kiesslich, R., Gossner, L., Goetz, M. Dahlmann, A., Vieth, M., Stolte, M., Hoffman, A., Jung, M., Nafe, B. Galle, P. R., and Neurath, M. F. (2006). In vivo histology of Barrett's esophagus and associated neoplasia by confocal laser endomicroscopy. Clin. Gastroenterol. Hepatol. 4, 979-987.

Leung, K. K., Maru, D., Abraham, S. Hofstetter, W. L., Mehran, R., and Anandasabapathy, S. (2009). Optical EMR: confocal endomicroscopytargeted EMR of focal high-grade dysplasia in Barrett's esophagus. Gastrointest. Endosc. 69, 170-172.
Liu, W., Hahn, H., Odze, R. D., and Goyal, R. K. (2009). Metaplastic esophageal columnar epithelium without goblet cells shows DNA content abnormalities similar to goblet cell-containing epithelium. Am. J. Gastroenterol. 104, 816-824.

Mannath, J., Subramanian, V., Kaye, P. V., and Ragunath, K. (2010). Life-threatening bleeding following Barrett's surveillance biopsies. Endoscopy 42, 211-212.

Neumann, H., Kiesslich, R., Wallace, M. B., and Neurath, M. F. (2010). Confocal laser endomicroscopy: technical advances and clinical applications. Gastroenterology 139, 388-392.

Neumann, H., Mönkemüller, K., Günther, C., Atreya, R., Vieth, M., and Neurath, M. F. (2012). Advanced endoscopic imaging for diagnosis of Crohn's disease. Gastroenterol. Res. Pract. 2012, 301541. 
Ormsby, A. H., Kilgore, S. P., Goldblum, J. R., Richter, J. E., Rice, T. W., and Gramlich, T. L. (2000). The location and frequency of intestinal metaplasia at the esophagogastric junction in 223 consecutive autopsies: implications for patient treatment and preventive strategies in Barrett's esophagus. Mod. Pathol. 13, 614-620.

Peters, F. P., Curvers, W. L., Rosmolen, W. D., de Vries, C. E., Ten Kate, F. J., Krishnadath, K. K., Fockens, P., and Bergman, J. J. (2008). Surveillance history of endoscopically treated patients with early Barrett's neoplasia: nonadherence to the Seattle biopsy protocol leads to sampling error. Dis. Esophagus 21, 475-479.

Pohl, H., Rösch, T., Vieth, M., Koch, M., Becker, V., Anders, M., Khalifa, A. C., and Meining, A. (2008). Miniprobe confocal laser microscopy for the detection of invisible neoplasia in patients with Barrett's oesophagus. Gut 57, 1648-1653.

Reid, B. J., Sanchez, C. A., Blount, P. L., and Levine, D. S. (1993). Barrett's esophagus: cell cycle abnormalities in advancing stages of neoplastic progression. Gastroenterology 105, 119-129.

Schlemper, R. J., Riddell, R. H., Kato, Y., Borchard, F., Cooper, H. S., Dawsey, S. M., Dixon, M. F., Fenoglio-Preiser, C. M., Fléjou, J. F., Geboes, K., Hattori, T., Hirota, T., Itabashi, M., Iwafuchi, M., Iwashita, A., Kim, Y. I., Kirchner, T., Klimpfinger, M., Koike, M., Lauwers, G. Y., Lewin, K. J., Oberhuber, G., Offner, F., Price, A. B., Rubio, C. A., Shimizu, M., Shimoda, T., Sipponen, P., Solcia, E., Stolte, M., Watanabe, H., and Yamabe, $H$.
(2000). The Vienna classification of gastrointestinal epithelial neoplasia. Gut 47, 251-255.

Sharma, P., Meining, A. R., Coron, E., Lightdale, C. J., Wolfsen, H. C., Bansal, A., Bajbouj, M., Galmiche, J. P., Abrams, J. A., Rastogi, A., Gupta, N., Michalek, J. E., Lauwers, G. Y., and Wallace, M. B. (2011). Realtime increased detection of neoplastic tissue in Barrett's esophagus with probe-based confocal laser endomicroscopy: final results of an international multicenter, prospective, randomized, controlled trial. Gastrointest. Endosc. 74, 465-472.

Tytgat, G. N. (1995). Does endoscopic surveillance in esophageal columnar metaplasia (Barrett's esophagus) have any real value? Endoscopy 27, 19-26.

Vieth, M. (2007). Low-grade dysplasia in Barrett's esophagus - an innocent bystander? Contra. Endoscopy 39, 647-649.

Vieth, M., and Barr, H. (2009). Editorial: defining a bad Barrett's segment: is it dependent on goblet cells? Am. J. Gastroenterol. 104, 825-827.

Vieth, M., Ell, C., Gossner, L., May, A., and Stolte, M. (2004). Histological analysis of endoscopic resection specimens from 326 patients with Barrett's esophagus and early neoplasia. Endoscopy 36, 776-781.

Vieth, M., and Stolte, M. (2000). Barrett's mucosa, Barrett's dysplasia and Barrett's carcinoma: diagnostic endoscopy without biopsy-taking does not suffice. Dis. Esophagus 13, 23-27.

Vieth, M., and Stolte, M. (2002). Barrett's esophagus and neoplasia: data from the Bayreuth Barrett's archive. Gastroenterology 122, 590-591.

Wallace, M., Lauwers, G. Y., Chen, Y., Dekker, E., Fockens, P., Sharma, P. and Meining, A. (2011). Miami classification for probe-based confocal laser endomicroscopy. Endoscopy 43, 882-891.

Wallace, M. B., Meining, A., Canto, M. I., Fockens, P., Miehlke, S., Roesch, T., Lightdale, C. J., Pohl, H., Carr-Locke, D., Löhr, M., Coron, E., Filoche, B. Giovannini, M., Moreau, J., Schmidt, C., and Kiesslich, R. (2010a). The safety of intravenous fluorescein for confocal laser endomicroscopy in the gastrointestinal tract. Aliment. Pharmacol. Ther. 31, 548-552.

Wallace, M. B., Sharma, P., Lightdale, C. Wolfsen, H., Coron, E., Buchner, A. Bajbouj, M., Bansal, A., Rastogi, A. Abrams, J., Crook, J. E., and Meining, A. (2010b). Preliminary accuracy and interobserver agreement for the detection of intraepithelial neoplasia in Barrett's esophagus with probe-based confocal laser endomicroscopy. Gastrointest. Endosc. 72, 19-24.

Wani, S., Falk, G. W., Post, J., Yerian, L., Hall, M., Wang, A., Gupta, N., Gaddam, S., Singh, M., Singh, V., Chuang, K. Y., Boolchand, V., Gavini, H., Kuczynski, J., Sud, P., Bansal, A. Rastogi, A., Mathur, S. C., Young, P., Cash, B., Goldblum, J., Lieberman, D. A., Sampliner, R. E., and Sharma, P. (2011). Risk factors for progression of low-grade dysplasia in patients with Barrett's esophagus. Gastroenterology 141, 1179-1186.

Winters, C. Jr., Spurling, T. J., Chobanian, S. J., Curtis, D. J., Esposito,
R. L., Hacker, J. F. III, Johnson, D. A., Cruess, D. F., Cotelingam, J. D., and Gurney, M. S. (1987). Barrett's esophagus. A prevalent, occult complication of gastroesophageal reflux disease. Gastroenterology 92 118-124.

Conflict of Interest Statement: Helmut Neumann: Speaker for Pentax, Mauna Kea Technologies, Essex Pharma, Abbott; research grants from Pentax, Siemens, Olympus, Nycomed, AstraZeneca, Pfizer. Cord Langner: none. Markus F. Neurath: Speaker, consultant, and advisory board member for Essex Pharma, MSD, Abbott, Giuliani, and Pentax. Research funding from the Deutsche Forschungsgemeinschaft. Michael Vieth: Speaker for Pentax, Mauna Kea Technologies, Altana, Falk, Astra Zeneca, Abbott, Olympus, Nycomed.

Received: 13 January 2012; paperpending published: 20 February 2012; accepted: 12 April 2012; published online: 07 May 2012.

Citation: Neumann H, Langner C, Neurath MF and Vieth M (2012) Confocal laser endomicroscopy for diagnosis of Barrett's esophagus. Front. Oncol. 2:42. doi: 10.3389/fonc. 2012.00042

This article was submitted to Frontiers in Gastrointestinal Cancers, a specialty of Frontiers in Oncology.

Copyright (c) 2012 Neumann, Langner, Neurath and Vieth. This is an open-access article distributed under the terms of the Creative Commons Attribution Non Commercial License, which permits noncommercial use, distribution, and reproduction in other forums, provided the original authors and source are credited. 